\title{
BMJ Open Complementary therapies for clinical depression: an overview of systematic reviews
}

\author{
Heidemarie Haller, ${ }^{\oplus}$ Dennis Anheyer, Holger Cramer, Gustav Dobos
}

To cite: Haller H, Anheyer D, Cramer $\mathrm{H}$, et al. Complementary therapies for clinical depression: an overview of systematic reviews. BMJ Open 2019;9:e028527. doi:10.1136/ bmjopen-2018-028527

- Prepublication history and additional material for this paper are available online. To view these files, please visit the journal online (http://dx.doi org/10.1136/bmjopen-2018028527).

Received 12 December 2018 Revised 18 June 2019 Accepted 08 July 2019
Check for updates

(C) Author(s) (or their employer(s)) 2019. Re-use permitted under CC BY-NC. No commercial re-use. See rights and permissions. Published by BMJ.

Department of Internal and Integrative Medicine, Evang. Kliniken Essen-Mitte, Faculty of Medicine, Universitat DuisburgEssen, Essen, Germany

Correspondence to

Dr Heidemarie Haller; h.haller@kem-med.com

\section{ABSTRACT}

Objectives As clinical practice guidelines vary widely in their search strategies and recommendations of complementary and alternative medicine (CAM) for depression, this overview aimed at systematically summarising the level 1 evidence on CAM for patients with a clinical diagnosis of depression.

Methods PubMed, Psyclnfo and Central were searched for meta-analyses of randomised controlled clinical trials (RCTs) until 30 June 2018. Outcomes included depression severity, response, remission, relapse and adverse events. The quality of evidence was assessed according to Grades of Recommendation, Assessment, Development, and Evaluation (GRADE) considering the methodological quality of the RCTs and meta-analyses, inconsistency, indirectness, imprecision of the evidence and the potential risk of publication bias.

Results The literature search revealed 26 meta-analyses conducted between 2002 and 2018 on 1-49 RCTs in major, minor and seasonal depression. In patients with mild to moderate major depression, moderate quality evidence suggested the efficacy of St. John's wort towards placebo and its comparative effectiveness towards standard antidepressants for the treatment for depression severity and response rates, while St. John's wort caused significant less adverse events. In patients with recurrent major depression, moderate quality evidence showed that mindfulness-based cognitive therapy was superior to standard antidepressant drug treatment for the prevention of depression relapse. Other CAM evidence was considered as having low or very low quality.

Conclusions The effects of all but two CAM treatments found in studies on clinical depressed patients based on low to very low quality of evidence. The evidence has to be downgraded mostly due to avoidable methodological flaws of both the original RCTs and meta-analyses not following the Consolidated Standards of Reporting Trials and Preferred Reporting Items for Systematic Reviews and Meta-Analyses guidelines. Further research is needed.

\section{INTRODUCTION}

Depression is one of the most prevalent psychiatric disorders, with about $25 \%$ of women and $12 \%$ of men suffering from at least one depressive episode during their lifetime. ${ }^{1-3}$ According to the criteria for diagnosis recommended by the American Psychiatric Association (APA), depressive disorders can

\section{Strengths and limitations of this study}

This systematic overview included the comprehensive literature search of important complementary and alternative medicine topics defined by the Cochrane Collaboration.

- The inclusion criteria were restricted to meta-analyses of randomised controlled clinical trials (RCTs) of patients with a clinical diagnosis of depression.

- The quality of evidence from meta-analyses was assessed according to Grades of Recommendation, Assessment, Development, and Evaluation.

- There is a possible lack of evidence of newer RCTs, which have not been analysed by the included meta-analyses.

be distinguished by their degree of severity or duration and are also characterised by a high comorbidity and an increase of psychological strain for the affected person. ${ }^{4}$ It is evident that a strong comorbid connection to several chronic conditions like addictions, ${ }^{5}$ neurodegenerative diseases ${ }^{67}$ or different psychiatric diseases $^{8-11}$ exists. This leads depressive disorders as one of the leading causes of disability worldwide. $^{12}$

The most commonly used treatments for depression are antidepressants, psychotherapy or a combination of drugs and psychotherapy. While both treatment strategies (alone and in combination) have been shown to be effective, ${ }^{13-15}$ more recent meta-analyses also found high dropout and low remission rates ${ }^{16-21}$ as well as clinically significant differences between antidepressant drugs and placebos only for patients at the upper end of the very severely depressed category. ${ }^{22}$ This may lead patients to search for alternatives. Increasing mainstream use of complementary and alternative medicine (CAM) support this trend, particularly for different physical conditions with comorbid affective disorders. ${ }^{23-27}$ The NIH defines CAM as therapeutic approaches that are usually not included in conventional Western medicine systems. ${ }^{28} \mathrm{CAM}$ therapies used in combination 
with conventional care are considered as complementary, those used instead of conventional care as alternative practices. Types of CAM approaches include natural products, such as herbs and dietary supplements (vitamins, minerals and probiotics) and mind and body practices, such as yoga, chiropractic and osteopathic manipulation, meditation, relaxation, acupuncture, tai chi, qi gong and hypnotherapy. Practices of traditional healers from Europe (naturopathy and homeopathy), Asia (Ayurveda and traditional Chinese medicine) and other continents are also classified as CAM. ${ }^{28}$ While some complementary therapies have become a promising adjunct in the standard treatment of depression, ${ }^{29} 30$ others are known for their possible side effects or interactions with standard drugs. ${ }^{30}$ Recent clinical practice guidelines, in addition, vary widely in their search strategies and resulting recommendations for CAM treatments. While the American College of Physicians (ACP) ${ }^{31}$ the American Psychiatric Association (APA $)^{32}$ and the Canadian Network for Mood and Anxiety Treatments (CANMAT) guideline ${ }^{33}$ provide a more comprehensive overview and critical appraisal of CAM treatments, the Deutsche Gesellschaft für Psychiatrie und Psychotherapie, Psychosomatik und Nervenheilkunde (DGPPN) ${ }^{34}$ the National Institute for Health and Care Excellence (NICE) ${ }^{35}$ and the World Federation of Societies of Biological Psychiatry (WFSBP) ${ }^{36}$ guidelines mainly focus on St. John's Wort and light therapy. Possible effects and risks of further CAM therapies are not discussed. Thus, the purpose of this overview is to provide a comprehensive search strategy of relevant CAM terms and systematically summarise the existing level 1 evidence for clinical depression as a basis for further guideline recommendations on the efficacy, effectiveness and safety of CAM therapies.

\section{METHODS}

This systematic overview of reviews was conducted in accordance with the Preferred Reporting Items for Systematic Reviews and Meta-Analyses (PRISMA) guidelines ${ }^{37} 38$ and the recommendations of the Cochrane Collaboration. ${ }^{39}$ The protocol was not prospectively registered.

\section{Patient and public involvement}

For this overview of reviews, patients or public were not involved.

\section{Inclusion and exclusion criteria}

- Types of studies: to be eligible, articles had to be systematic reviews with meta-analyses of randomised controlled trials (RCTs) published in peer-reviewed journals. Conference abstracts or unpublished work were excluded as well as reviews summarising evidence narratively. In cases of including same or similar original studies, only the review with the most recent, most comprehensive search was included. When systematic reviews reported results of RCTs as well as of designs of lower evidence levels, they were considered only if separate meta-analyses for the included RCTs were performed.

- Types of participants: only reviews of patients with a diagnosis of major depression or dysthymia were eligible as well as reviews including patients/general population samples with mild depressive symptoms above a clinical cut-off or seasonal patterns. In contrast, reviews studying depressive symptoms within specific subpopulations of substance-induced or demented patients, secondary depression due to another medical condition (eg, poststroke, cancer or pain patients), bipolar disorders, or females with premenstrual dysphoric disorder or postpartum depression were excluded. Further restrictions regarding the diagnostic criteria or procedures, regarding age, gender, duration of the condition or symptom intensity were not applied.

- Types of interventions: reviews investigating the effectiveness and/or safety of a single, adjunctive or combined CAM treatment were included. For the classification of CAM treatments the definition of the US National Institutes of Health ${ }^{40}$ was followed. CAM interventions have to be compared against treatment as usual (TAU)/waiting list, placebo/sham or standard medical care.

- Types of outcomes: reviews were eligible if they assessed at least one measure of effectiveness such as severity of depressive symptoms, response rate (generally defined as a $50 \%$ decrease in depression scores after a period of up to 12 weeks of treatment), ${ }^{31}$ remission rate (generally defined as a period of up to 12 weeks during which a patient is asymptomatic or has only few symptoms to a very mild degree),${ }^{41}$ relapse rates and/or a measure of safety such as number of adverse events (AEs), drug interactions or numbers needed to harm for study withdrawal due to side effects.

\section{Search strategy}

Electronic literature was systematically searched via PubMed, PsycInfo and Central from their inception to 31 January 2018, without restrictions regarding time or language. Search terms for CAM treatments were selected in accordance with Cochrane recommendations (table 1). ${ }^{42}$ Additional manual search included reference lists of previously published reviews $^{14293043}$ and clinical practice guidelines. ${ }^{31-36}$ Using PubMed Informer, ${ }^{44}$ the search was updated until 30 June 2018.

\section{Study selection process}

To assess eligibility, articles were selected by screening titles and abstracts independently by two authors (HH and DA). Any abstract considered potentially eligible by at least one author was read in full to decide on its eligibility. Disagreements were rechecked with a third author (HC) until consensus was achieved. 
Table 1 Electronic search strategy for PubMed

\#1 (Depression OR Depressive Disorder, Major OR Depressive Disorder, Treatment-Resistant OR Dysthymic Disorder OR Seasonal Affective Disorder)(mh)

\#2 (depress* OR dysthym* or "seasonal affective" OR "affective disorder" OR "affective disorders" OR "mood disorder" OR "mood disorders")(tiab)

\#3 (Systematic review OR Meta-analysis)(pt) OR (Systematic* OR Meta-analy*)(tiab)

\#4 (Acupressure OR Acupuncture OR acid OR Alexander Technique OR alternative OR Aromatherapy OR Aroma Therapy OR Art Therap* OR ayurved* OR Balneotherapy OR Balneo Therapy OR bee OR Biofeedback OR Bio Feedback OR bright light OR Chelation Therapy OR Chinese Traditional Medicine OR Chiropractic OR Chronotherapy OR Color Therapy OR complementary OR craniosacral OR cupping OR Curcumin OR Dance Therapy OR diet OR Distant Healing OR Electroacupuncture OR Feldenkrais OR folic acid OR Folate OR Healing Touch OR herbal OR Herbs OR Homeopath^ OR Honey OR Hydrotherapy OR hyperbaric oxygenation OR Hypericum perforatum OR Hyperthermia OR Hypnosis OR Imagery OR Inositol OR Kampo OR Light Therapy OR Magnesium OR Massage OR MBSR OR MBCT OR Meditation OR Mindfulness OR Morita Therapy OR Moxibustion OR Mediterranean OR Music Therap* OR Naturopathy OR Neural Therapy OR Omega-3 OR Osteopath* OR Ozone Therapy OR Phototherapy OR Pollen OR Prayer OR prebiotic* OR probiotic* OR Propolis OR Qi Gong OR Reflexology OR Reiki OR Relaxation OR Rolfing OR Royal Jelly OR S-adenosyl-L-methionine OR Saffron OR Shamanism OR Snoezelen OR Speleotherapy OR Spinal Manipulation OR Spiritual OR St John's Wort OR Supplements OR Tai Chi OR TCM OR Therapeutic Touch OR Traditional Chinese Medicine OR Tryptophan OR Tui na OR Turmeric OR vegan OR vegetarian OR venom OR Vitamin OR Yoga OR zinc)(tiab)

\#5 (Acupressure OR Acupuncture OR Acupuncture Therapy OR Acids OR Aromatherapy OR Art Therapy OR Balneology OR Biofeedback, Psychology OR Chelation Therapy OR Chiropractic OR Chronotherapy OR Color Therapy OR Complementary Therapies OR Crocus OR Curcuma OR Dance Therapy OR Diet OR Electroacupuncture OR Fatty Acids, Omega-3 OR Folic Acid OR Homeopathy OR Honey OR Hydrotherapy OR Hypericum OR Hyperthermia, Induced OR Hypnosis OR Imagery OR Inositol OR Magnesium OR Manipulation, Spinal OR Massage OR Medicine, Ayurvedic OR Medicine, Chinese Traditional OR Medicine, Kampo OR Meditation OR Mind-Body Therapies OR Mindfulness OR Moxibustion OR Naturopathy OR Ozone OR Phototherapy OR Plants, Medicinal OR Pollen OR Prebiotics OR Probiotics OR Propolis OR Qigong OR Relaxation OR Shamanism OR Speleotherapy OR Spiritual Therapies OR Supplements, Dietary OR Tai Ji OR Therapeutic Touch OR Tryptophan OR Venoms OR Vitamins OR Yoga OR Zinc)(mh)

\section{Data extraction and quality assessment}

Two authors (HH and DA) independently extracted data on the characteristics of the reviews including the type of the intervention, the year of publication, the number and quality of the original RCTs, the total number and age of the participants and effectiveness and safety outcomes. The quality of the included reviews was assessed using the Assessment of the Methodological Quality of Systematic Reviews (AMSTAR) tool. ${ }^{45}$ The AMSTAR tool consists of 11 items asking about important methodological quality criteria of systematic reviews such as: a published a priori design, duplicate study selection and data extraction, a comprehensive literature search including grey literature, a list of included and excluded studies, summarised characteristics and quality assessment of included studies, assessment of publication bias, appropriate method of data syntheses and deducing conclusions and a conflict of interests statement. AMSTAR has shown good construct validity and inter-rater reliability. The Intraclass Correlation Coefficient (ICC) of the total AMSTAR score of 11 points was reported as $0.84 .^{46}$ For this analysis, the two authors ( $\mathrm{HH}$ and DA) who independently assessed AMSTAR reached an ICC of 0.96. Disagreements regarding content or quality of the reviews were rechecked with a third author (HC) and resolved by agreement.

\section{Data synthesis}

Results were pooled qualitatively by type of the intervention. Outcomes had to be calculated as standard mean differences (SMDs), risk ratios (RRs), HRs or ORs. If meta-analyses displayed mean differences, SMDs were calculated using Review Manager Software (RevMan, V.5.3, The Nordic Cochrane Centre, Copenhagen, Denmark) for better comparability of the results. RevMan was also used to exclude SMDs/RRs/HRs/ORs of selective RCTs that did not fulfil eligibility criteria of this overview. Effect sizes were classified according to Cohen as SMDs of 0.2-0.49=small effect, SMDs of 0.5-0.79=medium effect and SMDs of $>0.8=$ large effect (absolute values) ${ }^{47}$ with higher reduction of/improvement in depression scores represented by more negative SMDs or RRs/HRs/ ORs less than 1. According to the NICE guideline, an SMD of $\geq 0.5$ or $\leq-0.5$, respectively, was considered as a clinically relevant reduction of depression severity. ${ }^{48}$ Statistical heterogeneity between studies was assessed by the $\chi^{2}$ test with a $\mathrm{p}$ value of $\leq 0.10$ indicating significant heterogeneity. The magnitude of heterogeneity was categorised by the $\mathrm{I}^{2}$ statistic with $\mathrm{I}^{2}$ of $0 \%-24 \%=$ no heterogeneity, $\mathrm{I}^{2}$ of $25 \%-49 \%=$ moderate heterogeneity, $\mathrm{I}^{2}$ of $50 \%-74 \%=$ substantial heterogeneity and $\mathrm{I}^{2}$ of $75 \%-100 \%=$ considerable heterogeneity. ${ }^{39}$ 


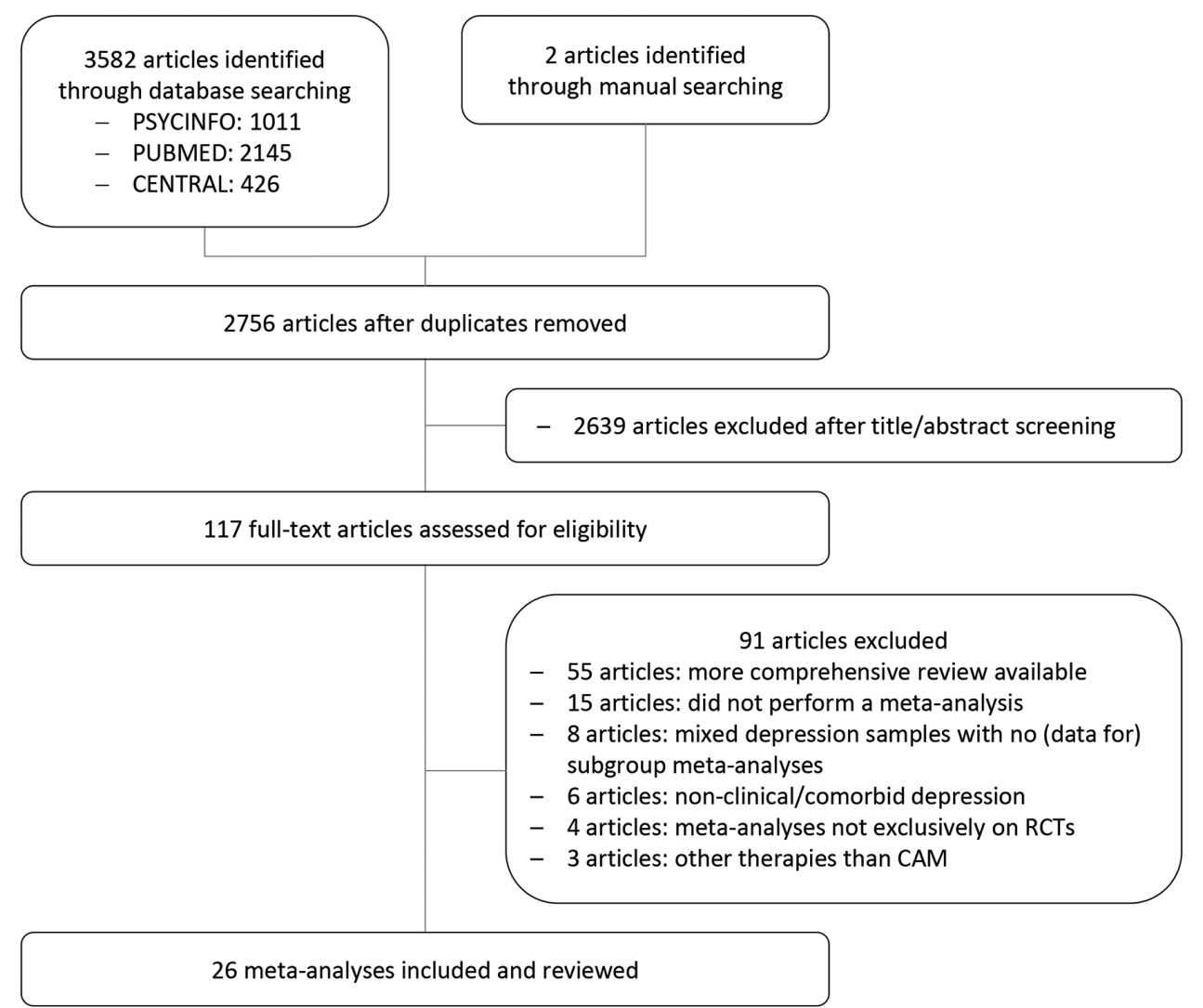

Figure 1 Study flow diagram. CAM, complementary and alternative medicine; RCTs, randomised controlled clinical trials.

\section{Quality of evidence}

The quality of evidence was assessed according to the Grades of Recommendation, Assessment, Development, and Evaluation (GRADE) approach ${ }^{49}$ individually by two authors (HH and DA). Disagreements were rechecked with a third author (HC) until consensus was achieved. For each outcome, the evidence can be graded as high, moderate, low or very low. Evidence from RCTs is initially assessed as high, but can be downgraded by one level for serious or two levels for very serious limitations of the study quality (of both RCTs and meta-analyses), inconsistency of the results, indirectness of the evidence, imprecision of the results and a potential risk of publication bias (as assessed by the included meta-analyses). ${ }^{49}$

\section{RESULTS}

\section{Study selection}

A total of 3582 potentially eligible articles were identified by electronic database search. One additional review was retrieved from manual search, ${ }^{50}$ one from the updated search until June 2018. ${ }^{51}$ After removing duplicates, 2639 articles were excluded by screening of titles and abstracts. The remaining 117 articles were read in full, of which further 91 reviews had to be excluded (figure 1). Reasons for exclusion comprised 55 reviews where newer and/or more comprehensive reviews on higher quality evidence were available. ${ }^{50-104}$ Further 15 reviews have to be excluded as they systematically summarised evidence but did not perform a meta-analysis mostly due to clinical heterogeneity or a limited number of available RCTs. ${ }^{105-119}$ Eight reviews were excluded as they included mixed depressive samples of bipolar or postpartum cases and did not provide (data for) subgroup analyses for patients with the defined depression criteria. ${ }^{120-127}$ Another six reviews contained community samples with non-clinical depression or physically ill patients with comorbid depressive symptoms but displayed no data for patients with a primary diagnosis of depression. ${ }^{128-133}$ Four reviews performed meta-analyses on both RCTs and non-RCTs and did not perform subgroup analyses or extracted sufficient data for post hoc analyses. ${ }^{134-137}$ Three of the reviews analysed standard instead of complementary therapies and were therefore excluded. Finally, 26 meta-analyses could be included and reviewed. ${ }^{138-163}$

\section{Review characteristics and quality}

Characteristics and quality appraisal of the included meta-analyses are summarised in the online supplementary table 1 . Meta-analyses were conducted between 2002 and 2018 and included between 1 and 49 RCTs on 40-7104 adult patients. Meta-analyses on children and adolescents were not available or did not meet inclusion criteria. Samples mostly consisted of patients suffering from major depressive disorder ${ }^{140-143} 145-151154156157159160$ but also included patients with mixed diagnoses of non-seasonal depression, ${ }^{138153162163}$ patients with a diagnosis of seasonal depression ${ }^{152}$ and patients with mild to severe symptoms of depression above a clinical cut-off. ${ }^{139} 141144145151155157158$ All but one meta-analysis ${ }^{141}$ reported pooled outcomes based 
on common standardised questionnaires or diagnostic interviews. Effects were analysed mostly up to 12 weeks of treatment (short term), except for four meta-analyses that included RCTs with effects reported up to 16,24 or 32 weeks ${ }^{138} 142143151160$ and further three meta-analyses with long-term analyses equal to or greater than 1 year. ${ }^{149} 157163$ The AMSTAR total scores of the included meta-analyses ranged between 4 and 11 points with a median quality of 7 points. The individual AMSTAR ratings are reported in the online supplementary table 2.

\section{Synthesis of results \\ Acupuncture \\ Manual acupuncture}

A high-quality Cochrane review meta-analysed 49 RCTs in major depressed adults as well as those with clinically relevant symptoms of depression for manual acupuncture. ${ }^{138}$ For depression severity, significant effect sizes were found in comparisons with TAU and as in adjunction to standard antidepressants, while acupuncture showed similar effects to invasive sham acupuncture and standard antidepressants (figure 2). The analyses of remission rates did not reveal superiority of acupuncture in the comparisons with TAU, invasive sham, standard antidepressants or in adjunction to standard antidepressants (figure 3). AEs reported in the acupuncture groups were significantly lower than in patients treated with antidepressant drugs. However, most meta-analyses showed significant heterogeneity, a lack of RCTs with low risk of bias and a possibly serious risk of publication bias. Thus, the quality of evidence had to be downgraded to low and very low.

\section{Electroacupuncture}

For electroacupuncture, the same Cochrane review ${ }^{138}$ revealed very low quality of evidence for the comparisons with TAU and invasive sham for both outcomes, severity

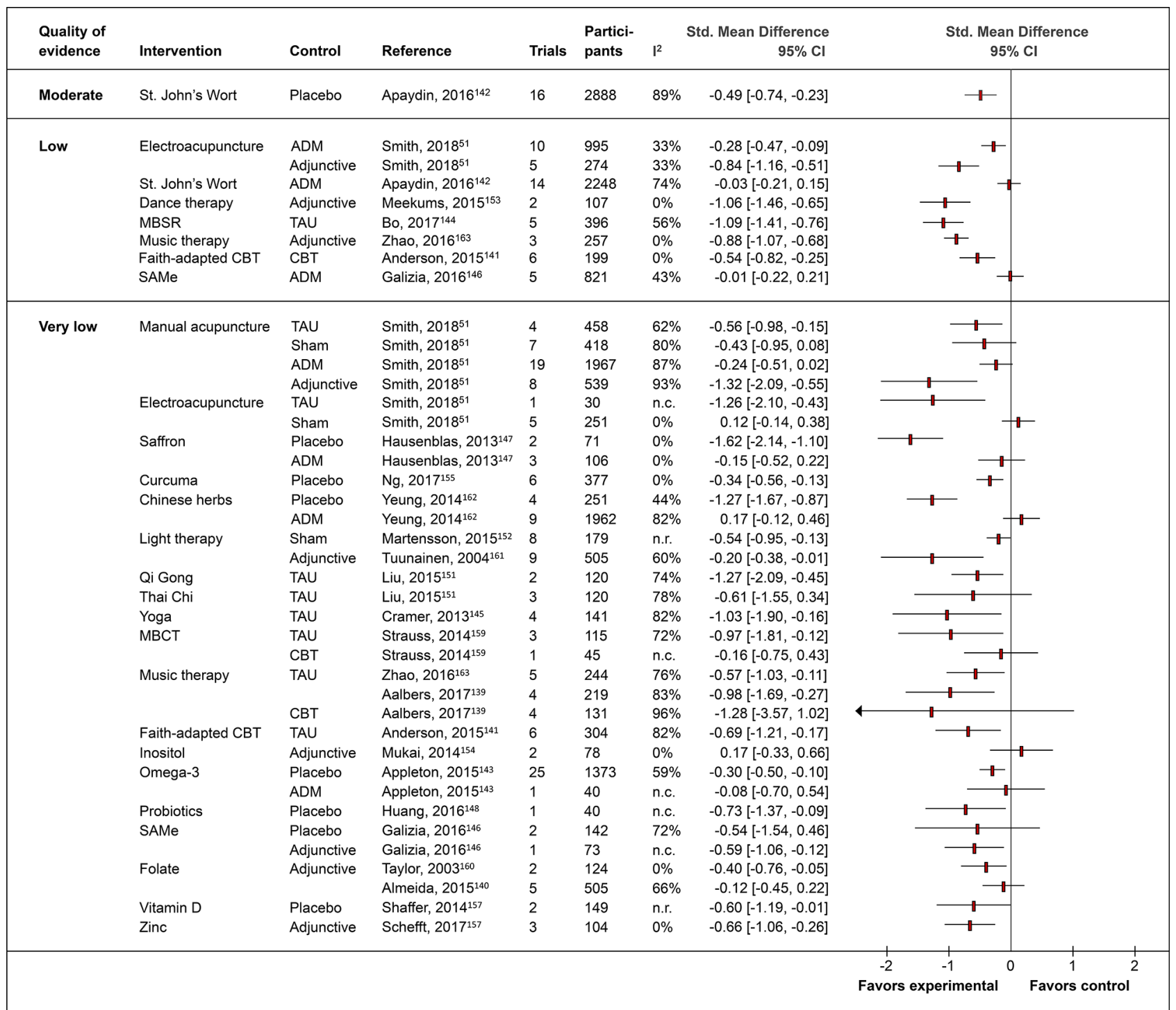

Figure 2 Quality of evidence for depression severity. ADM, antidepressant medication; CBT, cognitive-behavioural therapy; $\mathrm{I}^{2}$, heterogeneity; MBCT, mindfulness-based cognitive therapy; MBSR, mindfulness-based stress reduction; n.c., not calculable because of only one included randomised controlled clinical trials; n.r., not reported; SAMe, S-adenosyl methionine; TAU, treatment as usual. 


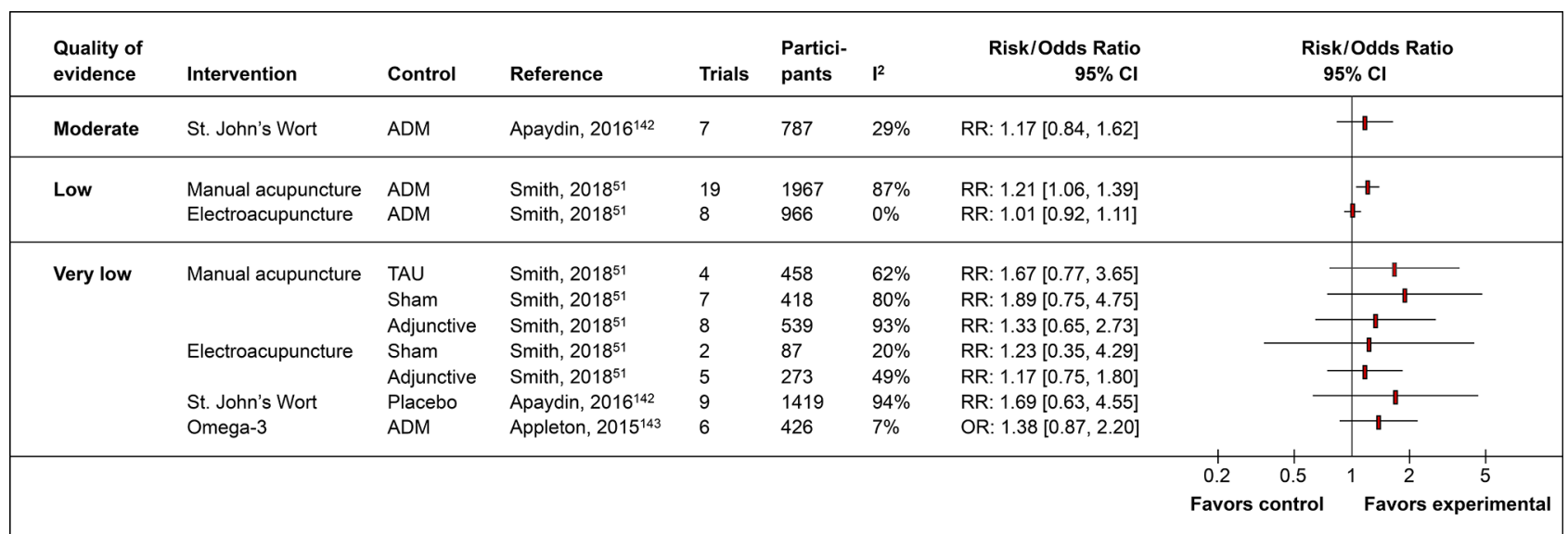

Figure 3 Quality of evidence for depression remission rates. ADM, antidepressant medication; $\mathrm{I}^{2}$, heterogeneity; RR, risk ratio; TAU, treatment as usual.

(figure 2) and remission (figure 3), because of serious limitations of the quality of the RCTs, imprecision and a high risk of publication bias. For electroacupuncture monotherapy in comparison with standard antidepressants, low-quality evidence homogeneously suggested significant greater effects for severity and similar effects for remission. As an adjunctive treatment to antidepressants, electroacupuncture effectiveness was supported by low quality of evidence showing a significant greater consistent and precise effect for depression severity. Although the mean adjunctive effect can be considered as large, the analysis based on only one RCT with overall low risk of bias and four RCTs of lower methodological quality that missed to include adjunctive sham acupuncture. For remission rates, very low quality of evidence suggested no effects in adjunction to antidepressants. In addition, one RCT showed significant less AEs when electroacupuncture was added to standard antidepressants.

\section{Aromatherapy}

The literature search revealed no meta-analysis on aromatherapy. A recent systematic review detected no RCTs in patients with a primary diagnosis of depression. However, two out of five of the reviewed studies on inhalation aromatherapy and five out of eight studies on aromatherapy massage have found significant antidepressive effects in mixed patient samples and healthy adults. ${ }^{116}$

\section{Biofeedback}

No meta-analysis on biofeedback was conducted to date. A recent systematic review revealed only one RCT on the defined inclusion criteria for depression showing some effects in contrast to sham psychotherapy. ${ }^{117}$

\section{Herbs}

\section{St. John's wort (Hypericum perforatum)}

The effectiveness of St. John's wort was meta-analysed by a Cochrane review of $29 \mathrm{RCTs}^{150}$ and a more recent, higher quality meta-analysis of 35 RCTs. ${ }^{142}$ In comparison with placebo, St. John's wort showed moderate quality evidence of significant greater reductions of depression severity (figure 2) and response rates (figure 4). The evidence had to be downgraded due to significant heterogeneity because of higher effects in studies from German-speaking countries than in those from the USA or other European countries. In contrast, very low quality of evidence suggested no superiority to placebo for remission (figure 3) and relapse rates (figure 5). In

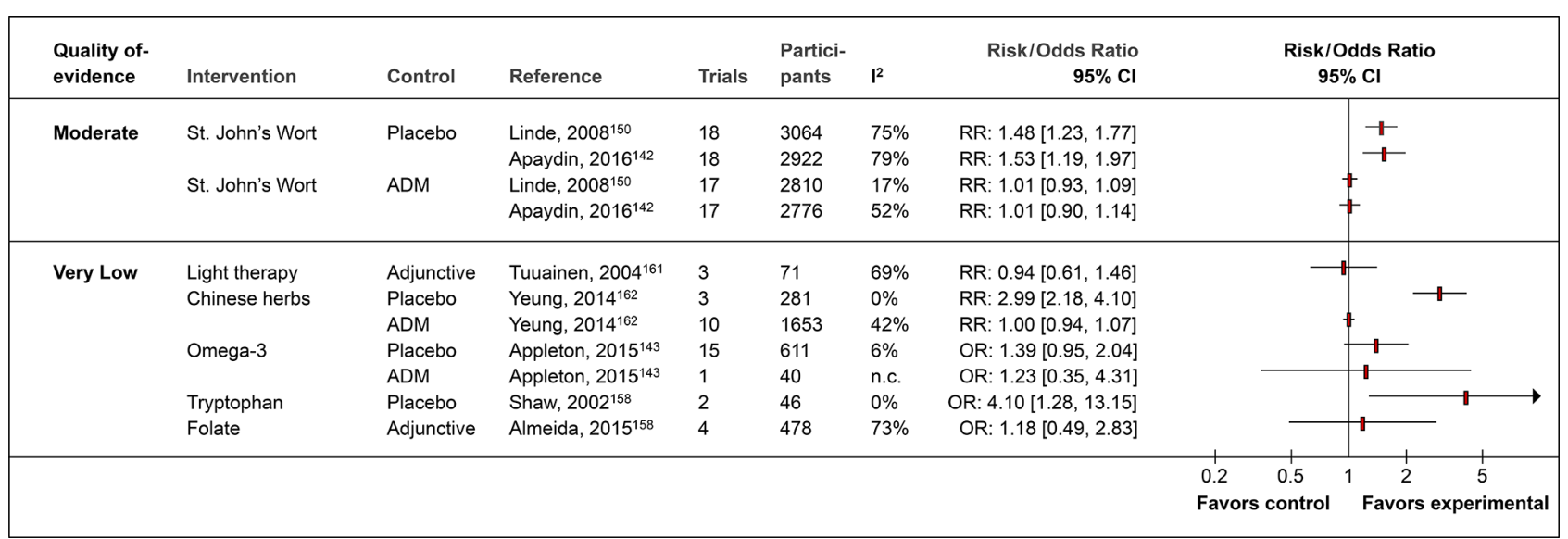

Figure 4 Quality of evidence for depression response rates. ADM, antidepressant medication; $I^{2}$, heterogeneity; n.c., not calculable because of only one included randomised controlled clinical trials; RR, risk ratio. 


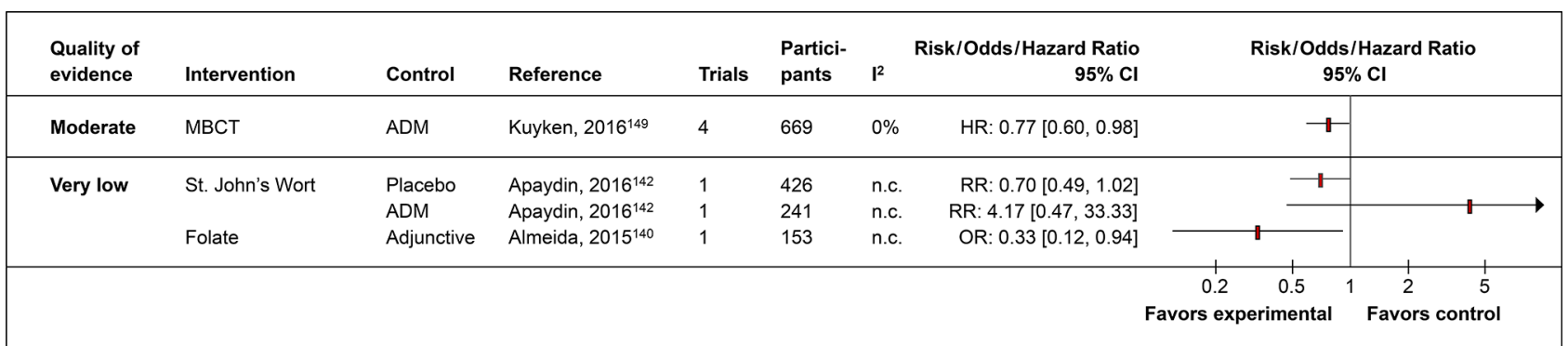

Figure 5 Quality of evidence for depression relapse rates. ADM, antidepressant medication; I $^{2}$, heterogeneity; MBCT, mindfulness-based cognitive therapy; n.c., not calculable because of only one included RCT; RR, risk ratio; TAU, treatment as usual.

comparison with standard antidepressants, St. John's wort showed comparable severity reductions, response, remission, and relapse rates. The quality of the evidence of the meta-analyses of severity and relapse rates had to be downgraded to low and very low, respectively. The evidence of the response and remission rates was considered as moderate quality showing the same results in both German and studies from other countries but containing some RCTs with unclear risk of selection bias and detection bias. Moreover, both meta-analyses ${ }^{142} 150$ showed similar AEs of St. John's wort to placebo but significant less AEs than standard antidepressants.

\section{Saffron (Crocus sativus)}

A moderate-quality meta-analysis examined the effectiveness and safety of saffron on depression severity by including five RCTs in adult patients with major depression. ${ }^{147}$ It revealed very low quality of evidence for significant greater effects versus placebo and similar effects versus antidepressant medication up to 8 weeks of treatment (figure 2). No serious AEs were reported, but patients receiving saffron tend to report more AEs than those receiving placebo and less AEs than those receiving antidepressant medication. Reasons for downgrading the evidence included no replication of the results (all included RCTs were conducted by the same research group), the small overall sample size and the possibly high risk of publication bias.

\section{Curcumin (Curcuma longa)}

For the intake of curcumin, a moderate-quality meta-analysis $^{155}$ revealed very low quality of evidence suggesting a small but significant short-term effect of low heterogeneity on depression severity by pooling six RCTs (figure 2). No serious AEs were recorded. Evidence had to be downgraded due to unclear risk of selection, performance, detection, attrition and reporting bias in over the half of the included RCTs, the imprecision of the pooled effect and the possibly high risk of publication bias.

\section{Traditional Chinese herbs}

A comprehensive but low-quality systematic review of 296 RCTs of Chinese herbal medicine formulas and single herbs ${ }^{162}$ revealed 21 RCTs of mostly unclear to high risk of selection, performance and detection bias and a serious risk of publication bias. Therefore, the evidence supporting the superiority above placebo and the similarity towards standard antidepressants regarding depression severity (figure 2) and response rates (figure 4) was assessed as very low.

\section{Other herbs}

For other than the described herbs, no meta-analyses were conducted to date. However, a systematic review ${ }^{109}$ found three single RCTs that showed significant improvement in depressive symptoms for Lavandula angustifolia as an adjunctive treatment to standard antidepressant drugs versus antidepressant drugs alone and for Echium amoenum and Rhodiola rosea versus placebo. No serious AEs were reported.

\section{Homoeopathy}

No meta-analysis on homoeopathic remedies for depression were conducted yet. A recent systematic review detected no placebo-controlled RCTs in patients with a primary diagnosis of depression. ${ }^{128}$

\section{Hypnosis}

No meta-analysis on hypnosis or self-hypnosis techniques met the inclusion criteria of this overview. The only available review on this topic ${ }^{126}$ included six RCTs among which only one RCT included adults with mild primary depression. Within the mixed sample of physically ill patients and healthy adults, (self-)hypnosis appeared to be effective in decreasing depressive symptoms.

\section{Light therapy}

A high-quality Cochrane review meta-analysed the effects of bright light therapy in adjunction to standard antidepressants versus sham light therapy plus antidepressants on severity and response rates in patients suffering from non-seasonal depression. ${ }^{161}$ By pooling 18 RCTs of overall unclear risk of bias, it revealed very low quality of evidence for a significant small but inconsistent and imprecise effect on depression severity (figure 2). A subgroup analysis of two RCTs with low risk of selection bias and detection bias revealed a significant large effect on depression severity but based on one non-peer-reviewed publication and one RCT that also included bipolar patients. Response rates did not significantly differ between 
groups (figure 4). AEs were reported non-systematically but appeared to be comparable with sham light therapy except for hypomania that occurred more often under verum light therapy. ${ }^{161}$

For patients with seasonal patterns of depression, a meta-analysis of eight RCTs ${ }^{152}$ revealed very low quality of evidence for a significant medium effect on depression severity of light monotherapy in comparison with sham light therapy (figure 2). Risk of bias of individual RCTs, heterogeneity and safety were not analysed leading to an overall low quality of the meta-analysis and downgrading of the evidence.

\section{Massage therapy}

The literature search detected no meta-analysis of massage therapy in patients with a primary depression. However, massage therapy appeared to be effective in decreasing depressive symptoms in mixed samples of physically ill patients and healthy adults. ${ }^{132}$ Future research will show whether these results may be transferable to primary depressed cases.

\section{Meditative movement therapies \\ Dance therapy}

Short-term effects of improvisatory or structured dance therapy as a combination of movement-based work, interactive group components and insight/expressive methods were meta-analysed by a Cochrane review of high methodological quality. ${ }^{153}$ It revealed a significant large pooled effect size for depression severity as an adjunctive treatment option to standard medical/psychotherapeutic care based on two RCTs (figure 2). Although the meta-analyses contained no heterogeneity and no imprecise CI, the evidence had to be downgraded to low quality because of mostly unclear/high risk of bias of one of the RCTs as well as the overall small sample size.

\section{Chinese movement therapies}

A low-quality meta-analysis on Chinese meditative movement therapies revealed 30 RCTs, of which 2 RCTs on Qi Gong and 3 RCTs on Tai Chi met inclusion criteria for patients with mild to severe symptoms of primary depression. ${ }^{151}$ Very low quality of evidence suggested significant short-term effects for Qi Gong but not for Tai Chi in comparison with TAU. The evidence had to be downgraded due to very serious limitations of the quality of the RCTs and the meta-analysis, significant heterogeneity, imprecision and a possible high risk of publication bias.

\section{Yoga}

A high-quality meta-analysis of complex yoga interventions for various depressive disorders found $12 \mathrm{RCTs},{ }^{145}$ of which 5 RCTs of mostly unclear risk of bias met the inclusion criteria of this overview. The pooled short-term effect on depression severity was of large size in comparison with TAU and medium size in comparison with standard exercises (figure 2). However, the evidence was assessed as very low due to serious limitations of quality of the RCTs, significant heterogeneity, imprecision and a possible high risk of publication bias.

A further systematic review of yoga in major depressive disorder, revealed five newer yoga RCTs but did not perform a meta-analysis because of high clinical heterogeneity. Risk of bias was comparably high and evidence mostly conflicting. ${ }^{106}$

\section{Mindfulness-based interventions}

Mindfulness-based cognitive therapy (MBCT)

A low-quality meta-analysis of mindfulness-based interventions in patients with major depression found four RCTs investigating the effects of MBCT and cognitivebehavioural therapy (CBT) on depression severity. ${ }^{159}$ It revealed a significant large short-term effect of MBCT in comparison with TAU and similar effects in comparison with CBT (figure 2). However, the quality of the evidence was considered as very low due to the missing risk of bias assessment, inconsistency and imprecision.

A further moderate-quality systematic review on MBCT meta-analysed 9 RCTs on an individual patient data level. ${ }^{149}$ The sample consisted of patients with recurrent major depression currently in remission. After a period of 60 weeks, MBCT showed a significantly reduced risk of depressive relapse compared with those receiving antidepressant drugs (figure 5). No serious AEs were reported. The evidence was assessed as moderate due to a possible risk of publication bias.

\section{Mindfulness-based stress reduction (MBSR)}

RCTs of MBSR and MBSR-like interventions were meta-analysed by a recent review ${ }^{144}$ showing a significant large short-term effect on depression severity in comparison with TAU and enhanced TAU (figure 2). The quality of evidence was assessed as low because of the overall unclear risk of selection and performance bias and significant heterogeneity.

\section{Music therapy}

Studies on active and receptive music therapy in older patients with a diagnosis of depression were summarised by a recent moderate-quality meta-analysis. ${ }^{163}$ Out of 19 RCTs, 8 met the inclusion criteria for this overview. Pooled analyses of five of them revealed a significant medium effect size on depression severity against TAU up to 52 weeks, however with bigger short-term than long-term effects, considerable heterogeneity and overall unclear risk of selection, performance and detection bias resulting in very low quality of evidence. Further three RCTs of the same review revealed low-quality evidence for a significant large consistent and precise effect of music therapy as an adjunctive treatment to antidepressants (figure 2).

A newer Cochrane review ${ }^{139}$ found eight different RCTs showing a significant large pooled effect of music therapy on depression severity against TAU and similar effects as CBT (figure 2). However, both analyses revealed very low quality of evidence due to mostly unclear selection, 
performance, detection and reporting bias, significant heterogeneity and imprecision.

\section{Nutrition therapy}

No meta-analyses on specific diets for patients with depression were published to date. A systematic review of 11 RCTs on whole-diet interventions in mostly healthy patients with subthreshold physical conditions revealed conflicting evidence on the effectiveness of those intervention for the reduction of depressive symptoms. ${ }^{114}$

A further systematic review on fasting in patients with chronic pain and inflammatory diseases ${ }^{110}$ included one RCT and seven observational studies, which showed promising short-term but questionable longer term antidepressive effects.

\section{Religious/spiritual interventions}

Very low to low quality of evidence was found by a moderate-quality systematic review of nine RCTs on Christian, Muslim and spiritual CBT adaptions. ${ }^{141}$ The analyses showed significant greater medium effects on depression severity against TAU and standard CBT (figure 2). Safety data were not reported.

\section{Supplements}

Inositol

A low-quality meta-analysis of two RCTs in patients with major depression ${ }^{154}$ revealed very low quality evidence for inositol as adjective to standard antidepressants versus placebo in combination to standard antidepressants (figure 2).

\section{Magnesium}

No meta-analysis of magnesium supplementation was found. A recent systematic review detected no RCTs in patients with a primary diagnosis of depression. ${ }^{107}$

\section{Omega-3 fatty acids}

A high-quality Cochrane review ${ }^{143}$ of 26 RCTs found conflicting evidence of the effectiveness of supplementation with omega-3 fatty acids versus placebo in patients with major depression as depression severity significantly improved while response and remission rates did not so (figures 2-4). One additional RCT with a very low sample size showed similar effects of omega-3 fatty acids on severity and response rates in comparison with antidepressant drug treatment (figures 2 and 4). However, all meta-analyses were based on very low quality of evidence because of limitations of the study quality, significant heterogeneity, imprecision and a possibly high risk of publication bias.

\section{Probiotics}

The effectiveness of the supplementation with probiotics on depression severity was analysed by a moderate-quality meta-analysis of five RCTs, of which only one RCT with overall low risk of bias was carried out on patients with major depression. ${ }^{148}$ The analysis of the RCT revealed a significant medium but imprecise short-term effect in comparison with placebo (figure 2). This led to overall very low quality of evidence for probiotics supplementation.

\section{S-adenosyl methionine (SAMe)}

A high-quality Cochrane review ${ }^{146}$ of the effectiveness and safety of SAMe supplementation on depression severity revealed two RCTs of low risk of bias that showed no significant pooled effects for SAMe monotherapy versus placebo. One RCT, also of low risk of bias, showed a significant medium short-term effect as adjunctive to standard antidepressant medication, both for depression severity. Further five RCTs, which were rated as having overall unclear risk of bias, showed similar pooled effects of SAMe monotherapy on depression severity compared with standard antidepressant medication (figure 2). Original RCTs reported safety issues insufficiently. For all meta-analyses, the evidence was assessed as low to very low quality because of limitations of the study quality, heterogeneity, imprecision and a possibly high risk of publication bias.

\section{Tryptophan}

A moderate-quality Cochrane review found two RCTs investigating the effectiveness and safety of tryptophan supplementation on depression severity. ${ }^{158}$ Pooling the effects led to significant greater short-term response rates (figure 4) as well as significant more AEs in the tryptophan group than in the placebo group. The evidence was assessed as very low quality because of an unclear risk of detection, attrition and other bias, imprecision and a possible risk for publication bias.

\section{Vitamins}

For Vitamin B6, no meta-analysis was available. A systematic review without meta-analysis revealed two RCTs showing no significant effects when compared with placebo. ${ }^{119}$

Two further moderate to high-quality meta-analyses examined the effects of vitamin B9 (Folate) for major depressive patients. While a Cochrane review ${ }^{160}$ calculated a significant medium effect size of folate intake as an adjunctive intervention to standard drug treatment on depression severity, a more recent review ${ }^{140}$ revealed non-significant differences on severity and response rates (figures 2 and 3). In contrast, a long-term combined intake of Vitamin B6, B9 and B12 was found to be effective for relapse prevention after remission of symptoms as a result of one RCT (figure 5). ${ }^{140}$ However, all comparisons were based on very low quality of evidence mostly due to significant heterogeneity, imprecision and possible high risk of publication bias.

Another moderate-quality meta-analysis revealed evidence of the effectiveness of vitamin D intake on depression severity in comparison with placebo. ${ }^{157}$ The analysis of the two included RCTs revealed a significant medium short-term effect in favour of vitamin $\mathrm{D}$ in major depressed patients up to 8 weeks (figure 2). The quality of evidence was rated as very low due to limitations of the study quality, missing values of heterogeneity, 
imprecision, a high risk of publication bias as well as insufficient reporting of AEs.

Zinc

The effectiveness of zinc for major depression was meta-analysed by a low-quality review of three RCTs. ${ }^{156}$ It revealed a significant pooled short-term effect of medium size and low heterogeneity when zinc was taken as an adjunctive to standard antidepressant drug treatment (figure 2). However, the available evidence had to be assessed as very low as the meta-analysis did not perform risk of bias assessments and did not report AEs.

\section{DISCUSSION}

This systematic review provided a comprehensive overview of the evidence of CAM treatments for patients with a diagnosis or clinical symptoms of depression. Moderate-quality evidence suggested the efficacy, comparative effectiveness to standard antidepressants and safety of St. John's wort on depression severity and response rates. For remission and relapse rates, the evidence was conflicting and of lower quality. Moreover, moderate quality evidence showed that MBCT was superior to standard antidepressant drug treatment for the prevention of depression relapse in patients with recurrent major depression. Low-quality evidence suggested significant greater effects in favour of electroacupuncture in comparison with standard antidepressants alone and in adjunction to standard antidepressants for depression severity. For remission rates, low quality evidence revealed comparable effects of electroacupuncture and standard antidepressants. Further significant greater effects, which are based on low-quality evidence, were found for MBSR versus TAU, music therapy in adjunction to standard antidepressants, faith-adapted CBT versus CBT, and SAMe versus standard antidepressants. Other treatments such as manual acupuncture, aromatherapy, biofeedback, herbs (crocus sativus, curcuma longa, traditional Chinese herbs, lavandula angustifolia, echium amoenum and rhodiola rosea), homoeopathy, hypnosis, bright light therapy, massage, meditative movement therapies (dance therapy, Qi Gong, Tai Chi and yoga), whole-diet interventions, fasting and supplementation with inositol, magnesium, omega-3 fatty acids, probiotics, tryptophan, $\mathrm{B}$ and $\mathrm{D}$ vitamins and zinc were based on very low quality of evidence or no level 1 evidence.

The strengths of the review process included the comprehensive literature search based on a structured list of CAM specific topics, which had been operationalised for the Cochrane Collaboration. ${ }^{42}$ It therefore included evidence for more than the previously considered CAM approaches and provided systematic information where further high-quality studies are required. In addition, we only included results of RCTs of patients with a diagnosis of depression or clinical relevant depressive symptoms and excluded RCTs on samples with minor or secondary symptoms of depression by newly calculating effect sizes.
Finally, we rated AMSTAR and considered the quality of the meta-analyses as well when grading the quality of the evidence.

The conclusions derived from this overview are limited due to possibly missing evidence from newer RCTs, which have not been summarised by the included systematic reviews and meta-analyses. As it was not within the scope of this overview, we did not separately search for individual RCTs. We also did not include meta-analyses on studies of lower evidence levels, which may include bigger samples and may provide additional information about further possible treatment approaches. Moreover, we did not search online registries or conference proceedings for unpublished or ongoing meta-analyses, which may limit the conclusions. Another reason that limits the quality of evidence consists in the unsatisfactory methodological quality of some of the included meta-analyses. Although the methodological quality of the original RCTs might be acceptable, the bad reporting of some meta-analyses led to downgraded evidence. In particular, meta-analyses often missed to search for grey literature, cite excluded studies, adequately assess risk of bias of the original studies and report complete $\mathrm{I}^{2}$ statistics. As the latter are known to be unstable in meta-analyses with a small numbers of studies, ${ }^{164}$ calculating confidence intervals for $\mathrm{I}^{2}$ should be standard. Moreover, RCTs as well as meta-analyses often missed to systematically report on occurred AEs, which also limits the significance of the conclusions. In RCTs of non-pharmacological interventions, there is always a high or unclear risk of performance bias and possibly high placebo effects. As such, adding credible sham interventions, controlling for patients' expectances and performing of intention-to-treat analyses is indispensable. However, meta-analyses mostly did not systematically assess these issues. In meta-analyses of pharmacological interventions, the influence of industrial funding sources was often not adequately analysed. Here, subgroup analyses of studies having received no funding/ non-industrial funding versus those having received industrial funding are needed. Results of meta-analyses that missed to report funding issues completely should interpreted with caution. In general, it should be noticed that all evidence is based on short-term pooled effects, except for meta-analyses of St. John's wort, MBCT, music therapy and $\mathrm{B}$ and $\mathrm{D}$ vitamins that also provided longer term follow-up data.

Clinical recommendations for patients should follow the country-specific clinical practice guidelines considering the quality of evidence, the accessibility of the treatment, costs and the preferences of the patients. While the guidelines agree 3132 34-36 165166 that clinicians should select between either CBT or second-generation antidepressant drugs for the treatment of major depression, the restricted search strategy of some of the guidelines might limit their recommendations for CAM treatments. For patients who do reject or do not tolerate standard antidepressant drugs, one alternative treatment option may be St. John's wort. It is also recommended by the 
American Psychiatric Association Task Force report ${ }^{43}$ and the CANMAT Depression Work Group ${ }^{33}$ as being proven sufficiently for the short-term by placebo-controlled and equivalence trials with standard antidepressants for mild to moderate major depression. Particularly for bridging the gap between diagnosis and getting access to psychotherapy and meanwhile reducing/not-worsening depression severity, St. John's wort may be considered as a possibly better tolerated alternative to standard antidepressant drugs. ${ }^{167}$ As St. John's wort is accessible without prescription and currently not regulated by the US Food and Drug Administration, we agree with the ACP guidelines ${ }^{31}$ that it remains difficult for patients to obtain quality-controlled remedies. Moreover, St. John's wort is associated with numerous herb-to-drug interactions. ${ }^{168}$ Therefore, we would recommend clinicians to educate their patients about possible effects, side effects and interactions who in turn should not take St. John's wort without professional advise. ${ }^{34}$ Despite those limitations, we would not discourage a general therapeutic attempt with St. John's wort, even if we disagree with the NICE guideline in this point. ${ }^{35}$ Clinicians may also inform patients with recurrent major depression currently in remission about the superiority of MBCT in comparison with standard antidepressants for relapse prevention. ${ }^{32-35}$ Finally, patients should also be informed that many other CAM treatments might show promising effects but cannot be recommended until further higher quality studies will confirm their effectiveness and safety.

Further research is needed, particularly for interventions that have shown preliminary evidence for reducing secondary symptoms of depression, promising short-term but no longer term effects or insufficient evidence due to low methodological quality of the original RCTs and/or the performed meta-analyses. Reporting of clinical trials and meta-analyses should necessarily follow the Consolidated Standards of Reporting Trials (CONSORT) ${ }^{169}$ and PRISMA guidelines, ${ }^{37}$ respectively, including rigorous documentation and analysis of AEs. Especially Chinese and Indian trials are still found to be poorly reported and tended to present more positive conclusions than those from western countries. ${ }^{170}{ }^{171}$ Moreover, seven of the included meta-analyses showed no more than poor methodological quality. All were published in peer-reviewed journals in the past 5 years. One complementary journal is among them, while six of the journals are conventional psychiatric journals with impact factors ranging from 2.419 to 4.369 . Thus, particularly, the review process as well as the editorial work need to be improved. Further clinical practice guidelines should extend their search strategies and include standard search terms for CAM. This is also important for CAM therapies that do not show consistent evidence or that are not yet investigated. This information might be equally interesting for physicians as well as for patients to make an informed decision about the treatment for clinical depression.

\section{CONCLUSION}

This overview of systematic reviews on CAM treatments for clinical depression aimed to provide a systematic search strategy and evidence base, on which further clinical practice guidelines may build their recommendations. To improve quality of trials and meta-analyses, researchers, reviewers as well as editors are asked to ensure that future articles strictly adhere the CONSORT and PRISMA guidelines.

Contributors HH was responsible for the conception and design of the study, the collection and analysis of the study data and for drafting the manuscript. DA participated in the analysis of the study data and drafting the manuscript. HC participated in the conception and design of the study and the analysis of the study data and critically revised the manuscript. GD participated in the conception and design of the study and critically revised the manuscript. All authors approved the final manuscript.

Funding The authors have not declared a specific grant for this research from any funding agency in the public, commercial or not-for-profit sectors.

Competing interests None declared.

Patient consent for publication Not required.

Provenance and peer review Not commissioned; externally peer reviewed.

Data availability statement All data relevant to the study are included in the article or uploaded as supplementary information.

Open access This is an open access article distributed in accordance with the Creative Commons Attribution Non Commercial (CC BY-NC 4.0) license, which permits others to distribute, remix, adapt, build upon this work non-commercially, and license their derivative works on different terms, provided the original work is properly cited, appropriate credit is given, any changes made indicated, and the use is non-commercial. See: http://creativecommons.org/licenses/by-nc/4.0/.

\section{REFERENCES}

1. Kessler RC, Berglund P, Demler O, et al. The epidemiology of major depressive disorder: results from the National comorbidity survey replication (NCS-R). JAMA 2003;289:3095-105.

2. Moussavi S, Chatterji S, Verdes E, et al. Depression, chronic diseases, and decrements in health: results from the world health surveys. Lancet 2007;370:851-8.

3. Rubio JM, Markowitz JC, Alegría A, et al. Epidemiology of chronic and nonchronic major depressive disorder: results from the National epidemiologic survey on alcohol and related conditions. Depress Anxiety 2011;28:622-31.

4. American Psychiatric Association. Diagnostic and statistical manual of mental disorders. 5th ed (DSM-5). Arlington: American Psychiatric Publishing, 2013.

5. Lai HMX, Cleary M, Sitharthan T, et al. Prevalence of comorbid substance use, anxiety and mood disorders in epidemiological surveys, 1990-2014: a systematic review and meta-analysis. Drug Alcohol Depend 2015;154:1-13.

6. Herbert J, Lucassen PJ. Depression as a risk factor for Alzheimer's disease: Genes, steroids, cytokines and neurogenesis - What do we need to know? Front Neuroendocrinol 2016;41:153-71.

7. Riccelli R, Passamonti L, Cerasa A, et al. Individual differences in depression are associated with abnormal function of the limbic system in multiple sclerosis patients. Mult Scler 2016;22:1094-105.

8. Azar M, Pruessner M, Baer LH, et al. A study on negative and depressive symptom prevalence in individuals at ultra-high risk for psychosis. Early Interv Psychiatry 2018;12:900-906.

9. Chechko N, Kellermann T, Augustin M, et al. Disorder-specific characteristics of borderline personality disorder with cooccurring depression and its comparison with major depression: an $\mathrm{fMRI}$ study with emotional interference task. Neuroimage Clin 2016;12:517-25.

10. Chen M-H, Pan T-L, Hsu J-W, et al. Attention-Deficit hyperactivity disorder comorbidity and antidepressant resistance among patients with major depression: a nationwide longitudinal study. Eur Neuropsychopharmacol 2016;26:1760-7.

11. Ronconi JM, Shiner B, Watts BV. A meta-analysis of depressive symptom outcomes in randomized, controlled trials for PTSD. $J$ Nerv Ment Dis 2015;203:522-9. 
12. Global Burden of Disease Study 2013 Collaborators. Global, regional, and national incidence, prevalence, and years lived with disability for 301 acute and chronic diseases and injuries in 188 countries, 1990-2013: a systematic analysis for the global burden of disease study 2013. Lancet 2015;386:743-800.

13. Cuijpers $P$, van Straten $A$, Andersson $G$, et al. Psychotherapy for depression in adults: a meta-analysis of comparative outcome studies. J Consult Clin Psychol 2008;76:909-22.

14. Gartlehner G, Wagner G, Matyas N, et al. Pharmacological and nonpharmacological treatments for major depressive disorder: review of systematic reviews. BMJ Open 2017;7:e014912.

15. Cipriani A, Furukawa TA, Salanti G, et al. Comparative efficacy and acceptability of 21 antidepressant drugs for the acute treatment of adults with major depressive disorder: a systematic review and network meta-analysis. Lancet 2018;391:1357-66.

16. Mathew SJ, Charney DS. Publication bias and the efficacy of antidepressants. Am J Psychiatry 2009;166:140-5.

17. Pigott HE, Leventhal AM, Alter GS, et al. Efficacy and effectiveness of antidepressants: current status of research. Psychother Psychosom 2010;79:267-79.

18. Rief W, Nestoriuc $\mathrm{Y}$, Weiss $\mathrm{S}$, et al. Meta-Analysis of the placebo response in antidepressant trials. J Affect Disord 2009;118:1-8.

19. Turner EH, Matthews AM, Linardatos E, et al. Selective publication of antidepressant trials and its influence on apparent efficacy. $N$ Engl J Med Overseas Ed 2008:358:252-60.

20. Forneris CA, Nussbaumer B, Kaminski-Hartenthaler A, et al. Psychological therapies for preventing seasonal affective disorder. Cochrane Database Syst Rev 2015;46.

21. Gartlehner G, Nussbaumer B, Gaynes BN, et al. Second-Generation antidepressants for preventing seasonal affective disorder in adults. Cochrane Database Syst Rev 2015;58.

22. Kirsch I, Deacon BJ, Huedo-Medina TB, et al. Initial severity and antidepressant benefits: a meta-analysis of data submitted to the food and drug administration. PLoS Med 2008;5:e45.

23. Costanian $\mathrm{C}$, Christensen RAG, Edgell $\mathrm{H}$, et al. Factors associated with complementary and alternative medicine use among women at midlife. Climacteric 2017;20:421-6.

24. Henson JB, Brown CL, Chow S-C, et al. Complementary and alternative medicine use in United States adults with liver disease. $J$ Clin Gastroenterol 2017;51:564-70.

25. Rhee TG, Westberg SM, Harris IM. Complementary and alternative medicine in US adults with diabetes: reasons for use and perceived benefits. J Diabetes 2018;10:310-9.

26. Zhang Y, Dennis JA, Leach MJ, et al. Complementary and alternative medicine use among US adults with headache or migraine: results from the 2012 National health interview survey. Headache 2017;57:1228-42.

27. Bahall M, Prevalence BM. Prevalence, patterns, and perceived value of complementary and alternative medicine among cancer patients: a cross-sectional, descriptive study. BMC Complement Altern Med 2017;17:345

28. $\mathrm{NCClH}$. Complementary, Alternative, or Integrative Health: What's In a Name? 2018. Available: https://nccih.nih.gov/health/integrativehealth [Accessed 06 Jun 2019].

29. Luberto CM, White C, Sears RW, et al. Integrative medicine for treating depression: an update on the latest evidence. Curr Psychiatry Rep 2013;15:391.

30. Ravindran AV, da Silva TL. Complementary and alternative therapies as add-on to pharmacotherapy for mood and anxiety disorders: a systematic review. J Affect Disord 2013;150:707-19.

31. Qaseem A, Barry MJ, Kansagara D, et al. Nonpharmacologic versus pharmacologic treatment of adult patients with major depressive disorder: a clinical practice guideline from the American College of physicians. Ann Intern Med 2016;164:350-9.

32. APA. Practice guideline for the treatment of patients with major depressive disorder. Washington, DC: American Psychiatric Association, 2010.

33. Ravindran AV, Balneaves LG, Faulkner G, et al. Canadian network for mood and anxiety treatments (CANMAT) 2016 clinical guidelines for the management of adults with major depressive disorder: section 5. complementary and alternative medicine treatments. Can J Psychiatry 2016;61:576-87.

34. DGPPN, BÄK, KBV. Clinical practice guideline for unipolar depression [S3-Leitlinie/Nationale VersorgungsLeitlinie Unipolare Depression - Langfassung, 2 Auflage. Version 5], 2015.

35. National Collaborating Centre for Mental Health. Depression: the treatment and management of depression in adults (updated edition. Leicester and London UK: The British Psychological Society \& The Royal College of Psychiatrists, 2010.

36. Bauer M, Pfennig A, Severus E, et al. World Federation of societies of biological psychiatry (WFSBP) guidelines for biological treatment of unipolar depressive disorders, part 1: update 2013 on the acute and continuation treatment of unipolar depressive disorders. World J Biol Psychiatry 2013;14:334-85.

37. Moher D, Liberati A, Tetzlaff J, et al. Preferred reporting items for systematic reviews and meta-analyses: the PRISMA statement. BMJ 2009;339.

38. Panic N, Leoncini $\mathrm{E}$, de Belvis $\mathrm{G}$, et al. Evaluation of the endorsement of the preferred reporting items for systematic reviews and meta-analysis (PRISMA) statement on the quality of published systematic review and meta-analyses. PLoS One 2013;8:e83138.

39. Higgins JPT, Green S. Cochrane Handbook for systematic reviews of interventions version 5.1.0: the Cochrane collaboration, 2011. Available: http://handbook.cochrane.org

40. National Center for Complementary and Integrative Health. Complementary, Alternative, or Integrative Health: What's In a Name? 2016. Available: https://nccih.nih.gov/health/integrativehealth [Accessed 24 Jul 2017].

41. Keller MB. Remission versus response: the new gold standard of antidepressant care. J Clin Psychiatry 2004;65(Suppl 4):53-9.

42. Wieland LS, Manheimer E, Berman BM. Development and classification of an operational definition of complementary and alternative medicine for the Cochrane collaboration. Altern Ther Health Med 2011:17:50-9.

43. Freeman MP, Mischoulon D, Tedeschini E, et al. Complementary and alternative medicine for major depressive disorder: a metaanalysis of patient characteristics, placebo-response rates, and treatment outcomes relative to standard antidepressants. J Clin Psychiatry 2010;71:682-8.

44. Muin M, Fontelo P, Ackerman M. Pubmed Informer: monitoring MEDLINE/PubMed through e-mail alerts, SMS, PDA downloads and RSS feeds. AMIA Annu Symp Proc 2005.

45. Shea BJ, Grimshaw JM, Wells GA, et al. Development of AMSTAR: a measurement tool to assess the methodological quality of systematic reviews. BMC Med Res Methodol 2007;7:10.

46. Shea BJ, Hamel C, Wells GA, et al. AMSTAR is a reliable and valid measurement tool to assess the methodological quality of systematic reviews. J Clin Epidemiol 2009;62:1013-20.

47. Cohen J. Statistical power analysis for the behavoral sciences. Hillsdale: Lawrence Erlbaum Associates, 1988.

48. National Institute for Clinical Excellence. Depression: management of depression in primary and secondary care. clinical practice guideline no 23. London: National Institute for clinical excellence, 2004: 670 .

49. Brozek JL, Akl EA, Alonso-Coello P, et al. Grading quality of evidence and strength of recommendations in clinical practice guidelines. Part 1 of 3 . An overview of the grade approach and grading quality of evidence about interventions. Allergy 2009;64:669-77.

50. Stub T, Alræk T, Liu J. Acupuncture treatment for depression-A systematic review and meta-analysis. Eur J Integr Med 2011;3:e259-70.

51. Al-Karawi D, Al Mamoori DA, Tayyar Y. The role of curcumin administration in patients with major depressive disorder: mini meta-analysis of clinical trials. Phytother Res 2016;30:175-83.

52. Appleton KM, Rogers PJ, Ness AR. Updated systematic review and meta-analysis of the effects of $n-3$ long-chain polyunsaturated fatty acids on depressed mood. Am J Clin Nutr 2010;91:757-70.

53. Appleton KM, Sallis HM, Perry R, et al. $\omega-3$ Fatty acids for major depressive disorder in adults: an abridged Cochrane review. BMJ Open 2016:6:e010172

54. Asher GN, Gartlehner G, Gaynes BN, et al. Comparative benefits and harms of complementary and alternative medicine therapies for initial treatment of major depressive disorder: systematic review and meta-analysis. J Altern Complement Med 2017;23:907-19.

55. Bloch MH, Hannestad J. Omega-3 fatty acids for the treatment of depression: systematic review and meta-analysis. Mol Psychiatry 2012;17:1272-82

56. Cabral P, Meyer HB, Ames D. Effectiveness of yoga therapy as a complementary treatment for major psychiatric disorders: a meta-analysis. Prim Care Companion CNS Disord 2011;13:PCC.10r01068.

57. Chi I, Jordan-Marsh M, Guo M, et al. Tai chi and reduction of depressive symptoms for older adults: a meta-analysis of randomized trials. Geriatr Gerontol Int 2013;13:3-12.

58. Clarke K, Mayo-Wilson E, Kenny J, et al. Can non-pharmacological interventions prevent relapse in adults who have recovered from depression? A systematic review and meta-analysis of randomised controlled trials. Clin Psychol Rev 2015;39:58-70.

59. Cui Y-H, Zheng Y. A meta-analysis on the efficacy and safety of St John's wort extract in depression therapy in comparison with 
selective serotonin reuptake inhibitors in adults. Neuropsychiatr Dis Treat 2016;12:1715-23.

60. Galante J, Iribarren SJ, Pearce PF. Effects of mindfulness-based cognitive therapy on mental disorders: a systematic review and meta-analysis of randomised controlled trials. $J$ Res Nurs 2013;18:133-55.

61. Golden RN, Gaynes BN, Ekstrom RD, et al. The efficacy of light therapy in the treatment of mood disorders: a review and metaanalysis of the evidence. Am J Psychiatry 2005;162:656-62.

62. Gowda U, Mutowo MP, Smith BJ, et al. Vitamin D supplementation to reduce depression in adults: meta-analysis of randomized controlled trials. Nutrition 2015;31:421-9.

63. Goyal M, Singh S, Sibinga EMS, et al. Meditation programs for psychological stress and well-being: a systematic review and metaanalysis. JAMA Intern Med 2014:174:357-68.

64. Hofmann SG, Sawyer AT, Witt AA, et al. The effect of mindfulnessbased therapy on anxiety and depression: a meta-analytic review. $J$ Consult Clin Psychol 2010;78:169-83.

65. Jorm AF, Christensen H, Griffiths KM, et al. Effectiveness of complementary and self-help treatments for depression. Med $\mathrm{J}$ Aust 2002;176 Suppl(Suppl):S84-96.

66. Kim HL, Streltzer J, Goebert D. St. John's wort for depression: a meta-analysis of well-defined clinical trials. J Nerv Ment Dis 1999;187:532-8.

67. Klainin-Yobas P, Oo WN, Suzanne Yew PY, et al. Effects of relaxation interventions on depression and anxiety among older adults: a systematic review. Aging Ment Health 2015;19:1043-55.

68. Kou M-J, Chen J-X. Integrated traditional and Western medicine fo treatment of depression based on syndrome differentiation: a metaanalysis of randomized controlled trials based on the Hamilton depression scale. J Tradit Chin Med 2012;32:1-5.

69. Kraguljac NV, Montori VM, Pavuluri M, et al. Efficacy of omega-3 fatty acids in mood disorders - a systematic review and metaanalysis. Psychopharmacol Bull 2009;42:39-54.

70. Lai J, Moxey A, Nowak G, et al. The efficacy of zinc supplementation in depression: systematic review of randomised controlled trials. J Affect Disord 2012;136:e31-9.

72. Li G, Mbuagbaw L, Samaan Z, et al. Efficacy of vitamin D supplementation in depression in adults: a systematic review. J Clin Endocrinol Metab 2014;99:757-67.

72. Lin P-Y, Su K-P. A meta-analytic review of double-blind, placebocontrolled trials of antidepressant efficacy of omega- 3 fatty acids. $J$ Clin Psychiatry 2007;68:1056-61.

73. Linde K, Berner M, Egger M, et al. St John's wort for depression: meta-analysis of randomised controlled trials. Br J Psychiatry 2005;186:99-107.

74. Linde K, Mulrow CD, Berner M, et al. St John's wort for depression. Cochrane Database Syst Rev 2005;(2):CD000448.

75. Man C, Li C, Gong D, et al. Meta-Analysis of Chinese herbal Xiaoyao formula as an adjuvant treatment in relieving depression in Chinese patients. Complement Ther Med 2014;22:362-70.

76. Martins JG. Epa but not DHA appears to be responsible for the efficacy of omega-3 long chain polyunsaturated fatty acid supplementation in depression: evidence from a meta-analysis of randomized controlled trials. J Am Coll Nutr 2009;28:525-42.

77. Mocking RJT, Harmsen I, Assies J, et al. Meta-Analysis and metaregression of omega-3 polyunsaturated fatty acid supplementation for major depressive disorder. Transl Psychiatry 2016;6:e756.

78. Moyer CA, Rounds J, Hannum JW. A meta-analysis of massage therapy research. Psychol Bull 2004;130:3-18.

79. Nussbaumer B, Kaminski-Hartenthaler A, Forneris CA, et al. Light therapy for preventing seasonal affective disorder. Cochrane Database Syst Rev 2015;46

80. Piet J, Hougaard E. The effect of mindfulness-based cognitive therapy for prevention of relapse in recurrent major depressive disorder: a systematic review and meta-analysis. Clin Psychol Rev 2011;31:1032-40

81. Qin F, Wu X-A, Tang Y, et al. Meta-Analysis of randomized controlled trials to assess the effectiveness and safety of free and easy Wanderer plus, a polyherbal preparation for depressive disorders. $J$ Psychiatr Res 2011;45:1518-24.

82. Rahimi R, Nikfar S, Abdollahi M. Efficacy and tolerability of Hypericum perforatum in major depressive disorder in comparison with selective serotonin reuptake inhibitors: a meta-analysis. Prog Neuropsychopharmacol Biol Psychiatry 2009;33:118-27.

83. Ren Y, Zhu C, Wu J, et al. Comparison between herbal medicine and fluoxetine for depression: a systematic review of randomized controlled trials. Complement Ther Med 2015;23:674-84.

84. Röder C, Schaefer M, Leucht S. [Meta-analysis of effectiveness and tolerability of treatment of mild to moderate depression with St. John's Wort]. Fortschr Neurol Psychiatr 2004;72:330-43.
85. Sarris J, Murphy J, Mischoulon D, et al. Adjunctive nutraceuticals for depression: a systematic review and meta-analyses. Am J Psychiatry 2016;173:575-87.

86. Sarris J, Panossian A, Schweitzer I, et al. Herbal medicine for depression, anxiety and insomnia: a review of psychopharmacology and clinical evidence. Eur Neuropsychopharmacol 2011;21:841-60.

87. Smith CA, Hay PPJ. Acupuncture for depression. Cochrane Database Syst Rev 2005;(2):CD004046.

88. Sublette ME, Ellis SP, Geant AL, et al. Meta-Analysis of the effects of eicosapentaenoic acid (EPA) in clinical trials in depression. $J$ Clin Psychiatry 2011;72:1577-84

89. Taylor MJ, Carney SM, Goodwin GM, et al. Folate for depressive disorders: systematic review and meta-analysis of randomized controlled trials. J Psychopharmacol 2004;18:251-6.

90. Wang C, Bannuru R, Ramel J, et al. Tai chi on psychological wellbeing: systematic review and meta-analysis. BMC Complement Altern Med 2010;10.

91. Wang F, Lee E-KO, Wu T, et al. The effects of tai chi on depression, anxiety, and psychological well-being: a systematic review and meta-analysis. Int J Behav Med 2014;21:605-17.

92. Wang $\mathrm{H}$, Qi H, Wang B-song, et al. Is acupuncture beneficial in depression: a meta-analysis of 8 randomized controlled trials? J Affect Disord 2008;111:125-34.

93. Wang Y, Fan R, Huang X. Meta-Analysis of the clinical effectiveness of traditional Chinese medicine formula Chaihu-Shugan-San in depression. J Ethnopharmacol 2012;141:571-7.

94. Wang $\mathrm{Y}-\mathrm{Y}$, Li X-H, Zheng W, et al. Mindfulness-based interventions for major depressive disorder: a comprehensive meta-analysis of randomized controlled trials. J Affect Disord 2018;229:429-36.

95. Whiskey E, Werneke U, Taylor D. A systematic review and metaanalysis of Hypericum perforatum in depression: a comprehensive clinical review. Int Clin Psychopharmacol 2001;16:239-52.

96. Yeung W-F, Chung K-F, Ng K-Y, et al. A meta-analysis of the efficacy and safety of traditional Chinese medicine formula Ganmai Dazao decoction for depression. J Ethnopharmacol 2014;153:309-17.

97. Yin J, Dishman RK. The effect of tai chi and Qigong practice on depression and anxiety symptoms: a systematic review and metaregression analysis of randomized controlled trials. Ment Health Phys Act 2014;7:135-46.

98. Zhang X, Kang D, Zhang L, et al. Shuganjieyu capsule for major depressive disorder (MDD) in adults: a systematic review. Aging Ment Health 2014;18:941-53.

99. Zheng W, Zhang YF, Zhong HQ, et al. Wuling capsule for major depressive disorder: a meta-analysis of randomised controlled trials. East Asian Arch Psychiatry 2016;26:87-97.

100. Ng QX, Venkatanarayanan N, Ho CYX. Clinical use of Hypericum perforatum (St John's wort) in depression: a meta-analysis. J Affect Disord 2017;210:211-21.

101. Chan Y-Y, Lo W-Y, Yang S-N, et al. The benefit of combined acupuncture and antidepressant medication for depression: a systematic review and meta-analysis. J Affect Disord 2015;176:106-17.

102. Smith CA, Hay PP, Macpherson $\mathrm{H}$, et al. Acupuncture for depression. Cochrane Database Syst Rev 2010;(1).

103. Zhang Y, Qu S-shan, Zhang J-ping, et al. Rapid onset of the effects of combined selective serotonin reuptake inhibitors and electroacupuncture on primary depression: a meta-analysis. $J$ Altern Complement Med 2016;22:1-8.

104. Zhang Z-J, Chen H-Y, Yip K-chee, et al. The effectiveness and safety of acupuncture therapy in depressive disorders: systematic review and meta-analysis. J Affect Disord 2010;124:9-21.

105. Coelho HF, Boddy K, Ernst E. Massage therapy for the treatment of depression: a systematic review. Int J Clin Pract 2008;62:325-33.

106. Cramer $\mathrm{H}$, Anheyer $\mathrm{D}$, Lauche $\mathrm{R}$, et al. A systematic review of yoga for major depressive disorder. J Affect Disord 2017;213:70-7.

107. Derom M-L, Sayón-Orea C, Martínez-Ortega JM, et al. Magnesium and depression: a systematic review. Nutr Neurosci 2013; $16: 191-206$.

108. Dolle K, Schulte-Körne G. [Complementary treatment methods for depression in children and adolescents]. Prax Kinderpsychol Kinderpsychiatr 2014;63:237-63.

109. Dwyer AV, Whitten DL, Hawrelak JA. Herbal medicines, other than St. John's wort, in the treatment of depression: a systematic review. Altern Med Rev 2011;16:40-9.

110. Fond $\mathrm{G}$, Macgregor $\mathrm{A}$, Leboyer $\mathrm{M}$, et al. Fasting in mood disorders: neurobiology and effectiveness. A review of the literature. Psychiatry Res 2013;209:253-8.

111. Hausenblas HA, Heekin K, Mutchie HL, et al. A systematic review of randomized controlled trials examining the effectiveness of saffron (Crocus sativus L.) on psychological and behavioral outcomes. $J$ Integr Med 2015;13:231-40. 
112. Jorm AF, Allen NB, O'Donnell CP, et al. Effectiveness of complementary and self-help treatments for depression in children and adolescents. Med J Aust 2006;185:368-72.

113. Maratos A, Gold C, Wang X, et al. Music therapy for depression. Cochrane Database Syst Rev 2008;25.

114. Opie RS, O'Neil A, Itsiopoulos C, et al. The impact of whole-of-diet interventions on depression and anxiety: a systematic review of randomised controlled trials. Public Health Nutr 2015;18:2074-93.

115. Pilkington $\mathrm{K}$, Kirkwood $\mathrm{G}$, Rampes $\mathrm{H}$, et al. Homeopathy for depression: a systematic review of the research evidence. Homeopathy 2005;94:153-63.

116. Sánchez-Vidaña DI, Ngai SP-C, He W, et al. The effectiveness of Aromatherapy for depressive symptoms: a systematic review. Evidence-Based Complementary and Alternative Medicine 2017;2017:1-21.

117. Schoenberg PLA, David AS. Biofeedback for psychiatric disorders: a systematic review. Appl Psychophysiol Biofeedback 2014;39:109-35.

118. Tsang HWH, Chan EP, Cheung WM. Effects of mindful and nonmindful exercises on people with depression: a systematic review. Br J Clin Psychol 2008;47:303-22.

119. Williams A-L, Cotter A, Sabina A, et al. The role for vitamin B-6 as treatment for depression: a systematic review. Fam Pract 2005;22:532-7.

120. Al-Karawi D, Jubair $L$. Bright light therapy for nonseasonal depression: meta-analysis of clinical trials. J Affect Disord 2016;198:64-71.

121. Grosso G, Pajak A, Marventano S, et al. Role of omega-3 fatty acids in the treatment of depressive disorders: a comprehensive metaanalysis of randomized clinical trials. PLoS One 2014;9:e96905.

122. Hallahan B, Ryan T, Hibbeln JR, et al. Efficacy of omega-3 highly unsaturated fatty acids in the treatment of depression. $\mathrm{Br} \mathrm{J}$ Psychiatry 2016:209:192-201.

123. Ng QX, Peters C, Ho CYX, et al. A meta-analysis of the use of probiotics to alleviate depressive symptoms. J Affect Disord 2018:228:13-19.

124. Penders TM, Stanciu CN, Schoemann AM, et al. Bright light therapy as augmentation of pharmacotherapy for treatment of depression: a systematic review and meta-analysis. Prim Care Companion CNS Disord 2016;18. doi:10.4088/PCC.15r01906.

125. Perera S, Eisen R, Bhatt M, et al. Light therapy for non-seasonal depression: systematic review and meta-analysis. BJPsych Open 2016;2:116-26.

126. Shih M, Yang Y-H, Koo M. A meta-analysis of hypnosis in the treatment of depressive symptoms: a brief communication. Int $J$ Clin Exp Hypn 2009:57:431-42.

127. Spedding S. Vitamin D and depression: a systematic review and meta-analysis comparing studies with and without biological flaws. Nutrients 2014:6:1501-18.

128. Davidson JRT, Crawford C, Ives JA, et al. Homeopathic treatments in psychiatry: a systematic review of randomized placebo-controlled studies. J Clin Psychiatry 2011;72:795-805.

129. Ernst E. Bach flower remedies: a systematic review of randomised clinical trials. Swiss Med Wkly 2010;140:w13079.

130. Galante J, Galante I, Bekkers M-J, et al. Effect of kindness-based meditation on health and well-being: a systematic review and metaanalysis. J Consult Clin Psychol 2014;82:1101-14.

131. Gonçalves JPB, Lucchetti G, Menezes PR, et al. Religious and spiritual interventions in mental health care: a systematic review and meta-analysis of randomized controlled clinical trials. Psychol Med 2015;45:2937-49.

132. Hou W-H, Chiang P-T, Hsu T-Y, et al. Treatment effects of massage therapy in depressed people: a meta-analysis. J Clin Psychiatry 2010;71:894-901.

133. Joyce J, Herbison GP, Cochrane Common Mental Disorders Group. Reiki for depression and anxiety. Cochrane Database Syst Rev 2015;117.

134. Blanck P, Perleth $\mathrm{S}$, Heidenreich $\mathrm{T}$, et al. Effects of mindfulness exercises as stand-alone intervention on symptoms of anxiety and depression: systematic review and meta-analysis. Behav Res Ther 2018:102:25-35.

135. Jun JH, Choi T-Y, Lee JA, et al. Herbal medicine (GAN Mai dA Zao decoction) for depression: a systematic review and meta-analysis of randomized controlled trials. Maturitas 2014;79:370-80.

136. Lee TM, Chan CC. Dose-Response relationship of phototherapy for seasonal affective disorder: a meta-analysis. Acta Psychiatr Scand 1999;99:315-23.

137. Nelms JA, Castel L. A systematic review and meta-analysis of randomized and nonrandomized trials of clinical emotional freedom techniques (EFT) for the treatment of depression. Explore 2016:12:416-26.
138. Smith CA, Armour M, Lee MS, et al. Acupuncture for depression. Cochrane Database Syst Rev 2018:9.

139. Aalbers S, Fusar-Poli L, Freeman RE, et al. Music therapy for depression. Cochrane Database Syst Rev 2017;20.

140. Almeida OP, Ford AH, Flicker L. Systematic review and metaanalysis of randomized placebo-controlled trials of folate and vitamin B12 for depression. Int Psychogeriatr 2015;27:727-37.

141. Anderson N, Heywood-Everett S, Siddiqi N, et al. Faith-adapted psychological therapies for depression and anxiety: systematic review and meta-analysis. J Affect Disord 2015;176:183-96.

142. Apaydin EA, Maher AR, Shanman R, et al. A systematic review of St. John's wort for major depressive disorder. Syst Rev 2016;5:148.

143. Appleton KM, Sallis HM, Perry R, et al. Omega-3 fatty acids for depression in adults. Cochrane Database Syst Rev 2015;63.

144. Bo A, Mao W, Lindsey MA. Effects of Mind-body interventions on depressive symptoms among older Chinese adults: a systematic review and meta-analysis. Int J Geriatr Psychiatry 2017;32:509-21.

145. Cramer H, Lauche R, Langhorst J, et al. Yoga for depression: a systematic review and meta-analysis. Depress Anxiety 2013;30:1068-83.

146. Galizia I, Oldani L, Macritchie K, et al. S-Adenosyl methionine (same) for depression in adults. Cochrane Database Syst Rev 2016;145.

147. Hausenblas HA, Saha D, Dubyak PJ, et al. Saffron (Crocus sativus L.) and major depressive disorder: a meta-analysis of randomized clinical trials. J Integr Med 2013;11:377-83.

148. Huang R, Wang K, Hu J. Effect of probiotics on depression: a systematic review and meta-analysis of randomized controlled trials. Nutrients 2016;8:483.

149. Kuyken W, Warren FC, Taylor RS, et al. Efficacy of MindfulnessBased cognitive therapy in prevention of depressive relapse: an individual patient data meta-analysis from randomized trials. JAMA Psychiatry 2016;73:565-74.

150. Linde K, Berner MM, Kriston L, et al. St John's wort for major depression. Cochrane Database Syst Rev 2008;19.

151. Liu X, Clark J, Siskind D, et al. A systematic review and metaanalysis of the effects of Qigong and tai chi for depressive symptoms. Complement Ther Med 2015;23:516-34.

152. Mårtensson B, Pettersson A, Berglund L, et al. Bright white light therapy in depression: a critical review of the evidence. $J$ Affect Disord 2015;182:1-7.

153. Meekums B, Karkou V, Nelson EA, et al. Dance movement therapy for depression. Cochrane Database Syst Rev 2015;115.

154. Mukai T, Kishi T, Matsuda Y, et al. A meta-analysis of inositol for depression and anxiety disorders. Hum Psychopharmacol 2014;29:55-63.

155. Ng QX, Koh SSH, Chan HW, et al. Clinical use of curcumin in depression: a meta-analysis. J Am Med Dir Assoc 2017;18:503-8.

156. Schefft C, Kilarski LL, Bschor T, et al. Efficacy of adding nutritional supplements in unipolar depression: a systematic review and metaanalysis. Eur Neuropsychopharmacol 2017;27:1090-109.

157. Shaffer JA, Edmondson D, Wasson LT, et al. Vitamin D supplementation for depressive symptoms: a systematic review and meta-analysis of randomized controlled trials. Psychosom Med 2014;76:190-6.

158. Shaw KA, Turner J, Del Mar C, et al. Tryptophan and 5-hydroxytryptophan for depression. Cochrane Database Syst Rev 2002;12.

159. Strauss C, Cavanagh K, Oliver A, et al. Mindfulness-based interventions for people diagnosed with a current episode of an anxiety or depressive disorder: a meta-analysis of randomised controlled trials. PLoS One 2014;9:e96110.

160. Taylor MJ, Carney SM, Geddes J, et al. Folate for depressive disorders. Cochrane Database Syst Rev 2003;60.

161. Tuunainen A, Kripke DF, Endo T, et al. Light therapy for nonseasonal depression. Cochrane Database Syst Rev 2004;64.

162. Yeung W-F, Chung K-F, Ng K-Y, et al. A systematic review on the efficacy, safety and types of Chinese herbal medicine for depression. J Psychiatr Res 2014;57:165-75.

163. Zhao K, Bai ZG, Bo A, et al. A systematic review and meta-analysis of music therapy for the older adults with depression. Int $J$ Geriatr Psychiatry 2016;31:1188-98.

164. von Hippel PT. The heterogeneity statistic $I^{(2)}$ can be biased in smal meta-analyses. BMC Med Res Methodol 2015;15:35.

165. Parikh SV, Quilty LC, Ravitz P, et al. Canadian network for mood and anxiety treatments (CANMAT) 2016 clinical guidelines for the management of adults with major depressive disorder: section 2 . psychological treatments. Can J Psychiatry 2016;61:524-39.

166. Kennedy SH, Lam RW, Mclntyre RS, et al. Canadian network for mood and anxiety treatments (CANMAT) 2016 clinical guidelines for the management of adults with major depressive disorder: 
section 3. pharmacological treatments. Can J Psychiatry 2016:61:540-60.

167. Fava GA, Gatti A, Belaise C, et al. Withdrawal symptoms after selective serotonin reuptake inhibitor discontinuation: a systematic review. Psychother Psychosom 2015;84:72-81.

168. Mills E, Montori VM, Wu P, et al. Interaction of St John's wort with conventional drugs: systematic review of clinical trials. BMJ 2004;329:27-30.

169. Schulz KF, Altman DG, Moher D, et al. Consort 2010 statement: updated guidelines for reporting parallel group randomised trials. BMJ 2010;340:c332.
170. Cramer $\mathrm{H}$, Lauche $\mathrm{R}$, Langhorst $\mathrm{J}$, et al. Are Indian yoga trials more likely to be positive than those from other countries? A systematic review of randomized controlled trials. Contemp Clin Trials 2015;41:269-72.

171. Ma B, Chen Z-min, Xu J-ke, et al. Do the CONSORT and stricta checklists improve the reporting quality of acupuncture and moxibustion randomized controlled trials published in Chinese journals? A systematic review and analysis of trends. PLOS One 2016;11:e0147244. 